\title{
Recurrent Facial Palsy Due to High Jugular Bulb Dehiscence
}

This article was published in the following Dove Press journal:

Journal of Multidisciplinary Healthcare

\author{
Fahd Alkhamis' \\ Khalid Alhajri' \\ Danah Aljaafari' \\ Ali Alhashim' \\ Adnan Alsamarah ${ }^{2}$ \\ Abdulaziz Sharydah $\mathbb{D}^{2}$ \\ Osama Basheir' \\ Feras AISulaiman (1D) \\ Majed Alabdali' \\ 'Department of Neurology, King Fahad \\ Hospital of the University, Imam \\ Abdulrahman Bin Faisal University, \\ Dammam, Saudi Arabia; ${ }^{2}$ Department of \\ Radiology, King Fahad Hospital of the \\ University, Imam Abdulrahman Bin Faisal \\ University, Dammam, Saudi Arabia
}

Background: A high mega jugular Bulb is an enlarged and swollen upper portion of the internal jugular vein with a variation of its anatomical position among the population, mostly situated below the hypotympanum. Although most cases of jugular bulb diverticulum are asymptomatic, excessive pressure on the surrounding structures might cause various symptoms, most prominently vertigo, sensorineural hearing loss, or tinnitus.

Case Report: A middle-aged male who was referred to the Department of Neurology with a recurrent complaint of left-sided facial weakness associated with headache and vertigo for a period of 24 months. His symptoms were episodic, furthermore exacerbated by visiting high altitude sites. Non-resolving with conventional medical treatment. After thorough investigation, including preoperative and postoperative audiograms, neuroimaging, including computed tomography as well as magnetic resonance angiography, he was diagnosed to have right-sided superior mega jugular bulb as a causative factor. After surgical management, the patient improved significantly. At his regular follow-up in our clinic there were no exacerbations of his symptoms.

Conclusion: Among patients who present with recurrent non-resolving facial palsy in which no apparent causative factor is identified, high jugular bulb should be suspected and investigated. Comprehensive and detailed medical history is essential for raising the suspicion for the diagnosis. Such as the case presented eliciting high altitudes as the main precipitating factor. The diagnosis is clinically elusive, commonly obscured by other common diagnoses. Surgery is recommended if antihypertensive drugs do not show improvement.

Keywords: internal jugular vein, high jugular bulb, facial nerve palsy, high jugular bulb dehiscence, headache, surgery

\section{Background}

The jugular bulb's anatomical position may vary, and it may extend medially toward the petrous apex and laterally toward the tympanic membrane. ${ }^{1} \mathrm{~A}$ high mega jugular bulb is a dilated upper bulbous portion of the internal jugular vein, originating from the sigmoid sinus at the level of jugular foramen. Although its location and size may vary, in most of the recorded cases the jugular bulb is situated beneath the hypotympanum. High jugular bulb is identified when the upper part of the jugular bulb protrudes to the lower part of the tympanic annulus, extending to the middle ear or the basal turn of the cochlea. ${ }^{2}$ A mega jugular bulb indicates a large cross-sectional caliber of the jugular bulb. Regardless of its position, the right jugular bulb and internal jugular vein are significantly larger than those on the left in approximately $66 \%$ of the population. ${ }^{3}$ Moreover, a jugular bulb
Correspondence: Majed Alabdali Neurology Departments, King Fahad Hospital of the University - Imam Abdulrahman Bin Faisal University, PO Box 40236, Dammam, 31952, Kingdom of Saudi Arabia

Tel +966-534347474

Email mmalabdali@iau.edu.sa
Journal of Multidisciplinary Healthcare 2021:14 359-362 
diverticulum is characterized by its location within the extended jugular bulb wall, potentially causing pressure on the neighboring structures and causing various symptoms. ${ }^{4}$ Although there are several reported cases in which patients relayed tinnitus, vertigo, or hearing loss. Most cases of high jugular bulb are asymptomatic. The symptoms associated with a jugular bulb diverticulum are mainly associated with its location. Vertigo, tinnitus, and sensorineural hearing loss can occur with a medial extension of the high jugular bulb, toward the petrous apex. On the other hand, a lateral protrusion alongside the tympanic cavity can manifest as vascular tinnitus and conductive hearing loss. ${ }^{5}$ Moreover, high jugular bulb can mimic Menière's disease, which is accompanied by recurrent episodes of vertigo, as described in the literature. ${ }^{6} \mathrm{We}$ describe a different presentation of this interesting clinical phenomenon to help improve understanding it.

\section{Case Report}

A 40-year-old man referred to the Department of Neurology with the complaint of recurrent facial weakness over 24 months duration. This was mainly on the left side and precipitated by visiting places of high altitudes. Upon further history taking, he also reported having episodic vertigo and headache that was associated with his facial weakness for the same duration. His symptoms were persistent for several hours despite different medical treatments. There were no known exacerbating nor relieving factors other than high altitude sites. The patient's condition was gradually worsening with each attack, resulting in a decline of his ability to perform daily activities. Describing symptoms onset, the patient stated developing a left lower facial asymmetry noticed by himself and his family. The patient has grade II on the House-Brackmann facial paralysis scale. There was difficulty travelling by plane or performing his hobby of mountaineering due to the probability of it aggravating his symptoms. Upon examination of the cranial nerves, a mild weakness of the right sided facial nerve was noted. Investigating his clinical condition, mastoid computed tomography showed the main abnormality as asymmetrical jugular bulbs which was larger on the right and protruding into the right tympanic cavity. There was associated thinning of the bone surrounding the right jugular bulb and direct communication with the external auditory canal. The mastoid portion of the facial nerve directly contacted the right mega jugular bulb. These findings are consistent with right-sided high mega jugular bulb in addition to dehiscence of the

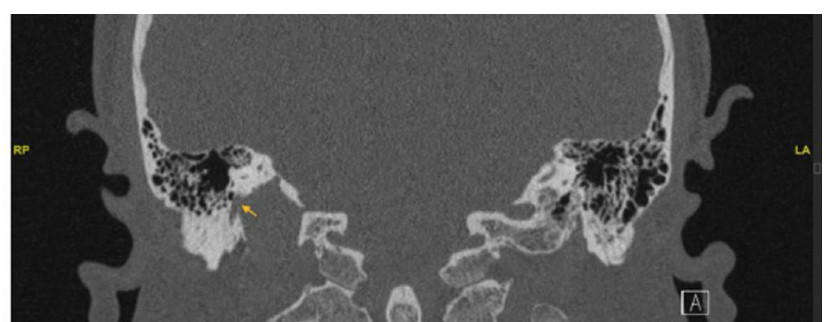

Figure I High resolution CT scan of the temporal bone in coronal plane obtained with thin slices of $1 \mathrm{~mm}$. The mastoid portion of the facial nerve shows direct communication with the right mega jugular bulb (yellow arrow), representing dehiscent ipsilateral facial nerve.

ipsilateral facial nerve and the posterior limb of the semicircular canal (Figures 1 and 2). Evidence of bilateral predominantly right-sided, superior semicircular canal dehiscence was found. These findings correlate well with the history of vertigo. Bilateral vestibular and cochlear aqueducts were symmetrical and within normal limits. Moreover, there was partial opacification of the left mastoid and bilateral sphenoid and maxillary air cells. The middle ear cavities including the incudomalleolar joint, aditus ad antrum, epi-tympanum, hypo-tympanum, and tegmen tympani were normal. However, the primary abnormality was seen on the right side, where a protrusion into the tympanic cavity was observed, with thinning of the bone surrounding the right jugular bulb.

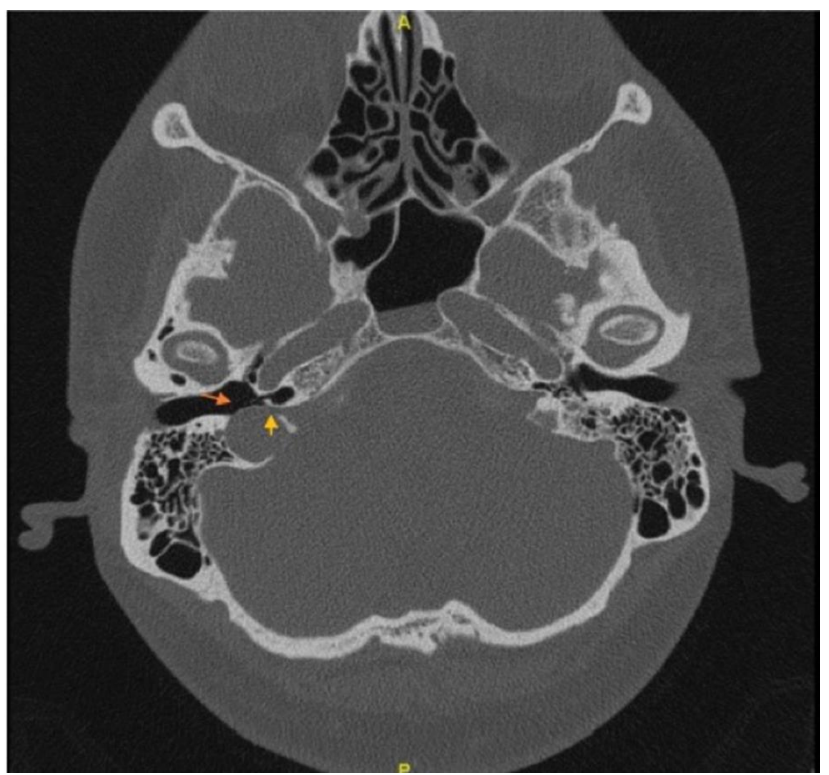

Figure 2 High resolution CT scan of the temporal bone in axial plane obtained with thin slices of I mm. It shows asymmetrical bilateral jugular bulbs, appears larger on the right side consistent with high mega jugular bulb, and slightly protruding into the tympanic cavity (red arrow), associated with bone thinning of bony outlines on the right jugular bulb. Also, illustrated direct communication with the external auditory canal (yellow arrow). 
A hearing test was performed with the result of unremarkable stapedial reflexes as well as tympanometry of normal range. A chronic neural lesion of the musculus orbicularis oris and musculus zygomaticus was revealed by performing a needle electromyoneurography. Displaying markedly decreased compound muscle conduction velocity in addition to decreased amplitude of the right facial nerve in comparison with the contralateral side. The patient was managed with Inderal 40mg taken twice a day. The patient provided written informed consent to publish his case details and accompanying images prior to writing the case. Institutional approval is not required to publish the case details.

\section{Discussion}

Although most anatomical jugular bulb variations and anomalies are asymptomatic, sometimes symptoms of aural fullness, unilateral hearing loss, or tinnitus may be present. In a case study reported in 1998, a patient with a dural malformation and no previous history of headaches later experienced a migraine-type headache. ${ }^{7}$ This is commonly found in patients with different vascular anomalies. On the other hand, symptoms involving the cranial nerves are rare. A reported case was found in the literature of an adult female suffering from petrous jugular malposition causing periodic facial palsy with hemifacial spasm in between her episodes since 16 years of age. She had recurrent headaches and lower facial weakness as well. However, surgery was not performed in that case. ${ }^{8}$ In another reported case of a female in her mid-30s with the same anomaly who underwent a trans-mastoid surgical procedure with a posterior tympanostomy, functional recovery was improved by facial nerve decompression. ${ }^{9}$ It is essential to investigate for any malignant neoplastic growth or any vascular anomaly in a patient with a suspicion of an enlarged jugular bulb or aberrant internal carotid artery. High-resolution computed tomography scan and magnetic resonance imaging are used to diagnose such cases. Computed tomography and digital angiography are frequently used to detect vascular pathology. The computed tomography scan in our patient showed an apparent high jugular bulb extending to the tympanic cavity reaching the external auditory canal. Absent osteolysis or damaged pneumatic cells. We postulate that by low barometric pressure the dilatation of the jugular bulb can increase, which in this case can aggravate facial weakness. Management is tailored to the patient's individual condition and presentation. ${ }^{10}$ Asymptomatic patients can be coincidentally discovered while investigating unrelated pathology, and treatment is not always required. If there is a planned ear surgery for any reason, it is important to assess for the presence of high jugular bulb. Rare cases of symptomatic involvement of the cranial nerves meet with surgical consideration. Competent venous drainage should be identified preoperatively. Surgery has low significance in patients who improved by antihypertensive drug such as the case presented. ${ }^{9}$

\section{Conclusion}

Although facial weakness is a common presentation to the neurology department and most commonly managed conservatively, rare causative etiology such as high jugular bulb should be investigated if the condition is recurrent. Patients with high jugular bulb can present with headache and facial palsy. Diagnosis is confirmed by neuroimaging. In the case presented, contact of the high jugular bulb with the mastoid segment of the facial nerve causes facial weakness. We highlight clues from medical history, that of low barometric pressure due to high altitudes might increase the dilatation of the jugular bulb. As a result, facial weakness increases. Antihypertensive agents are first line management, however, surgical intervention is recommended if symptoms are persistent.

\section{Consent}

Patient written informed consent was obtained to publish the case and accompanying images published.

\section{Disclosure}

The authorsdeclare that there are no conflicts of interest in this work.

\section{References}

1. Schmerber S, Lefournier V, Lavieille J-P, Boubagra K. Endolymphatic duct obstruction related to a jugular bulb diverticulum: high resolution CT and MR imaging findings. Clin Radiol. 2002;57(5):424-428. doi:10.1053/crad.2001.0919

2. Nayak S. Segmental anatomy of the temporal bone. Semin Ultrasound CT MR. 2001;22(3):184-218. doi:10.1016/S0887-2171(01)90007-1

3. Wadin $\mathrm{K}$, Wilbrand $\mathrm{H}$. The jugular bulb diverticulum. A radioanatomic investigation. Acta Radiol Diagn (Stockh). 1986;27(4):395-401. doi:10.1177/028418518602700405

4. Wadin K, Thomander L, Wilbrand H. Effects of a high jugular fossa and jugular bulb diverticulum on the inner ear. A clinical and radiologic investigation. Acta Radiol Diagn (Stockh). 1986;27(6):629-636. doi:10.1177/028418518602700603

5. Presutti L, Laudadio P. Jugular bulb diverticula. $O R L$ J Otorhinolaryngol Relat Spec. 1991;53(1):57-60. doi:10.1159/ 000276188 
6. Sterkers O, Bozorg Grayeli A, Julien N, Bouccara D, Rihane S, Chaigne P. [Jugular bulb diverticulum mimicking Menière's disease. Surgical treatment]. Ann Otolaryngol Chir Cervicofac. 1993;110 (7):363-371.

7. Adelman JU. Headaches and papilledema secondary to dural arteriovenous malformation. Headache. 1998;38(8):621-623. doi:10.1046/j.1526-4610.1998.3808621.x

8. Pappas DG, Hoffman RA, Cohen NL, Holliday RA, Pappas DG. Petrous jugular malposition (diverticulum). Otolaryngol Head Neck Surg. 1993;109(5):847-852. doi:10.1177/019459989310900512
9. Gal M, Darrouzet V, Pescio P, Vincey P, Bébéar JP. [Jugular bulb diverticular and facial paralysis]. Rev Laryngol Otol Rhinol (Bord). 1999;120(1):43-46.

10. Filipović B, Gjurić M, Hat J, Glunčić I. High mega jugular bulb presenting with facial nerve palsy and severe headache. Skull Base. 2010;20(6):465-468. doi:10.1055/s-0030-1261259

\section{Publish your work in this journal}

The Journal of Multidisciplinary Healthcare is an international, peerreviewed open-access journal that aims to represent and publish research in healthcare areas delivered by practitioners of different disciplines. This includes studies and reviews conducted by multidisciplinary teams as well as research which evaluates the results or conduct of such teams or healthcare processes in general. The journal covers a very wide range of areas and welcomes submissions from practitioners at all levels, from all over the world. The manuscript management system is completely online and includes a very quick and fair peer-review system. Visit http://www.dovepress.com/testimonials. php to read real quotes from published authors. 